\title{
Efectos del ácido butírico sobre la carcinogénesis experimental de colon inducida por 1,2-dimetilhidrazina en ratas
}

\author{
Catuogno, M.S.; Montenegro, M.A.; Ramírez, G.V.; Sánchez Negrette, M. \\ Cátedra Patología General y Sistemática, Facultad de Ciencias Veterinarias, \\ Universidad Nacional del Nordeste, Sargento Cabral 2139, Corrientes (3400), \\ Argentina. Tel-Fax: 54-379-4425753.E-mail: patgral@vet.unne.edu.ar.
}

\begin{abstract}
Resumen
Catuogno, M.S.; Montenegro, M.A.; Ramírez, G.V.; Sánchez Negrette, M.: Efectos del ácido butírico sobre la carcinogénesis experimental de colon inducida por 1,2-dimetilhidrazina en ratas. Rev. vet. 24: 1, 32-38, 2013. Estudios epidemiológicos en seres humanos vinculan a las dietas ricas en grasas con la aparición de cáncer de colon y recto. Paralelamente estudios experimentales con animales de laboratorio han determinado que no todas las grasas son nocivas para el organismo. Así, diversos componentes de la leche bovina, entre ellos el ácido butírico, son considerados potenciales inhibidores del proceso de la carcinogénesis. El objetivo de este trabajo consistió en evaluar los efectos anticancerígenos del ácido butírico sobre un modelo de carcinogénesis experimental del colon en ratas inducido con 1,2-dimetilhidrazina (DMH). Fueron utilizadas 110 ratas Wistar machos, con edad aproximada de 6 semanas $(150 \mathrm{~g})$, las cuales se dividieron al azar en grupos con y sin la administración diaria de ácido butírico y con inyecciones semanales de DMH durante 5 y 17 semanas. Los resultados obtenidos demostraron que la suplementación con ácido butírico en la dieta modifica el crecimiento de neoplasias colónicas provocando menor desarrollo del tamaño tumoral y menor frecuencia de neoplasias de comportamiento agresivo. Estos hechos corroboran que el desarrollo y comportamiento biológico del cáncer intestinal puede modificarse a través de la manipulación de la dieta.
\end{abstract}

Palabras clave: rata, ácido butírico, carcinogénesis, colon.

\begin{abstract}
Catuogno, M.S.; Montenegro, M.A.; Ramírez, G.V.; Sánchez Negrette, M.: Effect of butyric acid supplementation on experimental colon carcinogenesis induced by 1,2- dimethylhydrazine in rats. Rev. vet. 24: 1, 32-38, 2013. Epidemiological studies in human beings link fatty diets with the development of colon and rectum cancer. However, experimental studies on laboratory animals have determined that several components of bovine's milk fat such as butyric acid are considered to be potential inhibitors of carcinogenesis. The aim of this work was to study the anticancerigenous effect of butyric acid on an experimental carcinogenesis model of colon in rats induced by 1,2-dimethylhydrazine (DMH). One hundred and ten male Wistar 6 weeks old rats weighing $150 \mathrm{~g}$, were used. Animals were randomly separated in groups with and without butyric acid supplementation. They were divided also according to DMH weekly administration, in different experimental groups of 17 and 5 weeks respectively. The results support the fact that the development and biological behaviour of the colon cancer might be modified through diet changes.
\end{abstract}

Palabras clave: rat, butyric acid, carcinogenesis, colon.

\section{INTRODUCCIÓN}

De acuerdo a datos estadísticos aportados por la Asociación Americana del Cáncer, los decesos por cáncer en seres humanos ocupan el segundo lugar como causa de muerte luego de las enfermedades cardiovasculares ${ }^{3}$. Estudios epidemiológicos sugieren que el

Recibido: 30 julio 2012 / Aceptado: 9 octubre 2012
$35 \%$ de las muertes por cáncer son atribuibles a determinados tipos de dieta ${ }^{9}$. En este sentido la prevención a través de la dieta constituye una aproximación fácil y lógica para tratar y prevenir el cáncer ${ }^{14}$. En el ser humano las dietas ricas en grasas están vinculadas con la aparición de cáncer de colon y recto, mama, endometrio, próstata y pulmón. Las grasas involucradas son fundamentalmente las de origen animal, principalmente las saturadas, presentes en las carnes rojas y lácteos, 
así como las hidrogenadas, obtenidas a partir de aceites vegetales $^{21}$.

Es importante destacar que las poblaciones que consumen dietas con alto contenido de carne roja y grasa de origen animal, tienen mayor incidencia de cáncer de colon, explicando en parte el elevado porcentaje de esta afección en Argentina y Uruguay, históricamente grandes consumidores de carnes rojas ${ }^{11,25-27}$. En términos generales, las incidencias más altas ocurren Estados Unidos, Canadá, Australia, Dinamarca, Inglaterra, Uruguay y Argentina, asociadas a una dieta con alto contenido de grasas de origen animal, mientras que las tasas más bajas corresponden a los países de Asia, África y el resto de América del Sur, regiones con bajo consumo de carne y alta ingestión de frutas, vegetales y fibra ${ }^{5}$.

Por otra parte, estudios experimentales con animales de laboratorio han determinado que no todas las grasas son tan nocivas para el organismo, así diversos componentes de la leche bovina son considerados como potenciales inhibidores del proceso de la carcinogénesis, entre ellos la esfingomielina, el ácido linoleico conjugado, la vitamina $A$, el beta caroteno, la vitamina D y el ácido butírico $(\mathrm{AcBu})$. Una importante característica de los lípidos lácteos de los rumiantes es su alto contenido en ácido butírico. La grasa de la leche bovina contiene de 7 a 13 moles \% de ácido butírico en forma de butirildiacilgliceroles ${ }^{21}$. En tal sentido, nuestro grupo de investigación comprobó que la utilización de leche en polvo disminuyó significativamente la frecuencia de tumores intestinales inducidos con 1,2-dimetilhidrazina $(\mathrm{DMH})$ en ratas. Entre los componentes de la grasa láctea el ácido butírico jugaría un rol importante en dicha protección ${ }^{25}$.

Estudios en cultivos celulares permitieron discernir que el ácido butírico es un potente inductor de diferenciación in vitro para una amplia variedad de células neoplásicas, incluyendo leucemia, linfoma y cáncer de mama, colon, recto, hígado, cervix y ovario ${ }^{6}$. También induce apoptosis y posee efectos antiinflamatorios ${ }^{22}$. La inducción de la apoptosis es independiente del gen p53 y existen evidencias que ocurre como etapa final del proceso de diferenciación. El ácido butírico también bloquea la proliferación celular neoplásica al modificar la expresión de algunos genes implicados en el proceso de transmisión de señales: inhibe los protooncogenes ras, src y c-myc e induce la expresión de los protooncogenes $c$-fos y $c$-jun ${ }^{4}$. Las ciclinas, cinasas dependientes de ciclinas (cdk) y proteínas inhibidoras también forman parte de las vías moleculares por las cuales el ácido butírico causa detención del ciclo celular y/o diferenciación para prevenir la proliferación celular ${ }^{20}$. Además, juega un importante rol en la regulación del crecimiento celular, promueve la proliferación lenta de las células pero a la vez estimula la formación de criptas intestinales normales ${ }^{1,20,22,24}$.

Estudios experimentales utilizando butirato de sodio como fuente de ácido butírico, administrado a ratas vía oral e intracecalmente en altas concentraciones, de- mostraron efectos promotores en algunas investigaciones y efectos anticancerígenos en otras ${ }^{18}$. Intentando demostrar que el responsable del aumento de cáncer de colon en ratas es el sodio y no el butirato vía oral, se hicieron intentos suplementando con tributirina, con la cual no se obtuvieron diferencias en áreas focales de displasia o tumores colónicos ${ }^{8}$.

El objetivo del presente trabajo consistió en estudiar los efectos anticancerígenos del ácido butírico administrado en altas concentraciones, sobre un modelo de carcinogénesis del intestino grueso inducida experimentalmente en ratas.

\section{MATERIAL Y MÉTODOS}

Se utilizaron ratas Wistar machos, con edad aproximada de 6 semanas (150 g), las cuales permanecieron alojados en un ambiente climatizado $\left(21^{\circ} \mathrm{C}\right)$, en jaulas metálicas individuales, con agua y alimento balanceado comercial ad libitum. Para la inducción de tumores se utilizó el conocido cancerígeno 1,2-dimetilhidrazina (DMH). Se conformaron dos grupos: A (control sin $\mathrm{DMH}$ ) y B (tratados con DMH), grupos que se mantuvieron en recintos separados para evitar contaminaciones con el cancerígeno.

A su vez, el grupo A fue dividido en lote 1, constituido por 10 animales alimentados con un balanceado comercial y agua, y lote 2 , conformado por 20 ratas alimentadas con el mismo balanceado, cuya agua de bebida fue suplementada con ácido butírico puro $(4 \mathrm{mg} / \mathrm{ml})$.

El grupo B se subdividió en: lote 3 (20 animales tratados con 17 inyecciones de DMH y mantenidos con alimento balanceado y agua), lote 4 (20 animales tratados con 5 inyecciones de DMH y mantenidos con alimento balanceado y agua), lote 5 (20 animales tratados con 17 inyecciones de DMH, mantenidos con alimento balanceado y suplementados con ácido butírico en el agua de bebida) y lote 6 (20 animales tratados con 5 inyecciones de $\mathrm{DMH}$, mantenidos con alimento balanceado y suplementados con ácido butírico en el agua de bebida).

Inicialmente, como fase de adaptación, se suministraron a todos los lotes las dietas respectivas durante los 14 días previos al comienzo del tratamiento con DMH. La administración de ácido butírico continuó hasta el final del experimento. La droga DMH fue administrada mediante inyecciones subcutáneas a la dosis de 20 $\mathrm{mg} / \mathrm{kg}$ de peso, en forma semanal, durante 17 semanas a los animales de los lotes 3 y 5 , y durante 5 semanas a los lotes 4 y 6 .

En la preparación de la solución madre del cancerígeno se utilizó 1,2-dimetilhidrazina de la firma Sigma Chemical Co. (symetrical dimethylhydrazina dihydrochloride), la cual fue disuelta a razón de $400 \mathrm{mg} / 100 \mathrm{ml}$ de solución de agua destilada que contenía $37 \mathrm{mg} / 100$ ml de EDTA como agente estabilizador. Esta solución madre fue llevada a pH 6,5 mediante el agregado de 15 a 20 gotas de hidróxido de sodio 1 normal, que fue preparada cada semana, momentos antes de la inoculación. 
Para la preparación de la solución de ácido butírico se dispuso de tal sustancia con $99 \%$ de pureza $\left(\mathrm{C}_{4} \mathrm{H}_{8} \mathrm{O}_{2}\right.$ de la firma Aldrich), el cual fue administrado en la bebida a razón de $4 \mathrm{mg} / \mathrm{ml}$ de agua 14 días previos al tratamiento con DMH y se continuó hasta la finalización de la experiencia. Estas altas concentraciones de ácido butírico se previeron teniendo en cuenta que la leche de vaca contiene un promedio $0.959 \mathrm{mg} / \mathrm{ml}$ de dicho ácido y que una rata adulta bebe por día aproximadamente entre 20 y $30 \mathrm{ml}$ de agua (si bebiese leche de vaca estaría consumiendo diariamente ente 19 y $29 \mathrm{mg}$ de ácido butírico). La manipulación de las drogas se realizó con guantes y material descartable, con posterior eliminación apropiada de los mismos. El agua de bebida con el ácido butírico fue renovada diariamente siendo eliminado el sobrante del día anterior.

Las ratas fueron examinados diariamente anotando las alteraciones físicas y de comportamiento. El peso corporal fue registrado al inicio de la experiencia y semanalmente hasta el sacrificio de los animales. Todas las ratas fueron sacrificadas al concluir la semana número 26 desde el inicio de la experiencia. En la necropsia, el aparato digestivo completo (esófago, estómago, intestino delgado y colon) fue retirado para su examen macro y microscópico. El intestino grueso (ciego, colon proximal, colon distal y recto) fue incidido longitudinalmente realizando un corte sobre el lado opuesto a la inserción mesentérica, a fin de observar la mucosa y la eventual presencia de lesiones. Se analizó la existencia, localización, tamaño, forma y características de los tumores en cada una de las partes anatómicas. Todo el material obtenido se fijó en formol bufferado al $10 \%$ durante 24 horas. Posteriormente con cada parte anatómica se llevó a cabo la técnica de swiss-roll que consiste en remover el intestino del abdomen para luego dividirlo en segmentos permitiendo su más fácil manipulación. Cada segmento fue enrollado longitudinalmente sobre si mismo ${ }^{19}$, y luego fue procesado según la técnica histológica clásica para bloques parafinados, cortados en $5 \mu \mathrm{m}$ y coloreados con Hematoxilina Eosina (HE), PAS y Alcian Blue a pH 2,5.

Los tumores fueron clasificados macroscópicamente en polipoides y no polipoides. Los primeros se

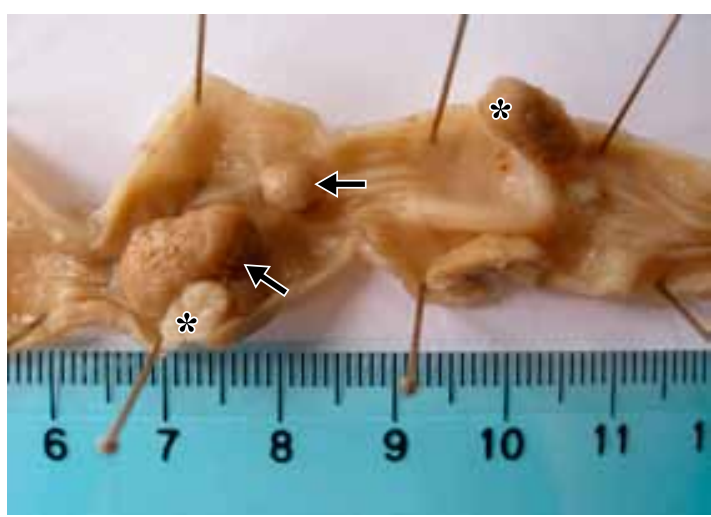

Figura 1. Adenocarcinomas en colon distal, de tipo polipoide con base pediculada (flechas) y polipoides con base sésil (asteriscos). caracterizan por marcada proliferación de la mucosa y crecimiento protuberante, mientras que los no polipoides o planos son lesiones que no superan dos veces el grosor de la mucosa, son deprimidos y en ocasiones ulcerados o con formas levemente elevadas ${ }^{17}$. A su vez, los polipoides se clasificaron en pediculados y sésiles. Los tumores pediculados son aquellos que están unidos a la mucosa por medio de un tallo o pedículo mientras que los sésiles no presentan pedículo y poseen una base de implantación ancha (mayor base que altura) insertada directamente en la pared del colon.

Para la identificación de las distintas partes anatómicas se recurrió a la descripción realizada por Lindström ${ }^{15}$. El análisis estadístico de la significancia de la cantidad de tumores por lote se realizó a través de un diseño no paramétrico (test de Kruskall-Wallis).

\section{RESULTADOS}

Para el estudio macroscópico del intestino grueso se tuvo en cuenta el número, tamaño, forma y localización de los tumores en cada uno de los segmentos que lo componen. En los lotes 1 y 2 no se observaron tumores.

Número de tumores. El mayor número de tumores se presentó en los lotes que recibieron el cancerígeno durante 17 semanas. En el lote 3 (DMH-17) se hallaron en total 166 tumores, con un promedio de 8,3 tumores por rata $(\mathrm{t} / \mathrm{r})$; el lote $5(\mathrm{AcBu}+\mathrm{DMH}-17)$ registró $142 \mathrm{tu}-$ mores (promedio: 7,47 t/r). El mayor número de tumores observados por rata en ambos lotes correspondió a un animal del lote 3 con 19 tumores (Figura 1) seguido por un animal del lote 5 con 16 tumores. Los ejemplares que recibieron suplementación con $\mathrm{AcBu}$ desarrollaron menor número de tumores. El lote 4 (DMH-5) presentó un total de 63 tumores, con un promedio de $3,15 \mathrm{t} / \mathrm{r}$, mientras que el lote $6(\mathrm{AcBu}+\mathrm{DMH}-5)$ registró 54 tumores con un promedio de 2,7 t/r. El mayor número de tumores observados por rata en ambos lotes correspondió a un animal del lote 40 con 9 tumores, seguido por una rata del lote 6 con 7 tumores. La suplementación con $\mathrm{AcBu}$ provocó menor desarrollo de tumores en los animales al compararlos con los que no recibieron el ácido graso.

Tamaño de los tumores. El tamaño de los tumores presentó variaciones de rango desde 1 a $5 \mathrm{~mm}, 6$ a 10 $\mathrm{mm}, 11$ a $15 \mathrm{~mm}, 16$ a $20 \mathrm{~mm}$ y más de $20 \mathrm{~mm}$ (Tabla 1). Los tumores que fueron diagnosticados microscópicamente se incluyeron en el rango hasta $5 \mathrm{~mm}$.

Todos los animales de los cuatro lotes tratados con DMH presentaron mayor porcentaje de tumores en el rango de 1 a 5 milímetros, sin encontrarse diferencias significativas entre los distintos lotes. No se observaron tumores de más de $16 \mathrm{~mm}$ en los lotes 4 y 5 . En el lote 6 no se visualizaron tumores mayores de $11 \mathrm{~mm}$. Los tumores de mayor tamaño fueron encontrados en el lote 3 , cuyos animales presentaron mayor número de inoculaciones de $\mathrm{DMH}$ y no ingirieron $\mathrm{AcBu}$.

En la Tabla 2 se muestran los diferentes tipos macroscópicos de tumores aparecidos y su presentación 
Tabla 1. Tamaño de los tumores registrados en ratas tratadas con DMH $(\mathrm{mm})$.

\begin{tabular}{lccccc}
\hline lote & $<5$ & $6-10$ & $11-15$ & $16-20$ & +20 \\
\hline $3(\mathrm{DMH}-17)$ & 125 & 34 & 5 & 1 & 1 \\
& $75,30 \%$ & $20,48 \%$ & $3,01 \%$ & $0,60 \%$ & $0,60 \%$ \\
$4(\mathrm{DMH}-5)$ & 47 & 14 & 2 & & - \\
$5(\mathrm{AcBu}+\mathrm{DMH}-17)$ & $74,60 \%$ & $22,22 \%$ & $3,17 \%$ & - & - \\
& 103 & 33 & 6 & & - \\
$6(\mathrm{AcBu}+\mathrm{DMH}-5)$ & $44 \%$ & $23,23 \%$ & $4,22 \%$ & - & - \\
\hline
\end{tabular}

Tabla 2. Tipos macroscópicos de tumores registrados en ratas tratadas con DMH.

\begin{tabular}{lccccc}
\hline lote & $\begin{array}{c}\text { polipoide } \\
\text { pediculado }\end{array}$ & $\begin{array}{c}\text { polipoide } \\
\text { sésil }\end{array}$ & plano & $\begin{array}{c}\text { ulcero- } \\
\text { infiltrante }\end{array}$ & total \\
\hline $3(\mathrm{DMH}-17)$ & 7 & 61 & 56 & 8 & 132 \\
& $5,30 \%$ & $46,21 \%$ & $42,42 \%$ & $6,06 \%$ & $132 \%$ \\
$4(\mathrm{DMH}-5)$ & 5 & 30 & 18 & 1 & 54 \\
$5(\mathrm{AcBu}+\mathrm{DMH}-17)$ & 12 & 70 & 30 & 6 & 118 \\
& $10,16 \%$ & $59,32 \%$ & $25,42 \%$ & $5,09 \%$ & 118 \\
$6(\mathrm{AcBu}+\mathrm{DMH}-5)$ & 4 & 31 & 15 & & 50 \\
\hline
\end{tabular}

en cada uno de los lotes. Los cuatro lotes tratados con DMH presentaron un mayor porcentaje de tumores de tipo polipoide con base sésil (Figura 1), siendo el lote 6 el que registró el porcentaje más elevado de este tipo de tumor (62\%). Por otra parte el lote 3 presentó el mayor porcentaje de tumores planos $(42,42 \%)$ y ulceroinfiltrantes $(6,06 \%)$. El lote 4 acusó una menor incidencia $(\mathrm{p}<0,001)$ de tumores de tipo ulceroinfiltrante, considerados como de mayor malignidad desde el punto de vista de su comportamiento biológico, por su capacidad infiltrante y metastatizante. En los animales suplementados con AcBu el desarrollo de neoplasias ulceroinfiltrantes fue menor, no presentándose ninguna neoplasia con estas características en el lote 6 .

Tipo histológico de las neoplasias. El estudio microscópico del intestino grueso consistió en la determinación e identificación de neoplasias y lesiones displásicas en sus diferentes partes anatómicas. Los tumores fueron clasificadas de acuerdo al tipo histoló- gico, considerándose también el grado de infiltración, la frecuencia de aparición y la localización de cada tipo. A la microscopía los tumores fueron clasificados en benignos y malignos. Los tipos benignos encontrados fueron adenoma tubular (AT) y adenoma tubulovelloso (ATV). Por otra parte los tipos malignos fueron adenocarcinoma simple (ACS), carcinoma de células en anillo de sello (CCas), carcinoma mucinoso (CMu) y carcinoma mixto (CMix). En la Tabla 3 se especifica la cantidad de tumores registrados en cada lote, según los tipos histológicos detallados ut supra. El ACS fue el tipo histológico predominante en todos los lotes tratados con DMH. El lote 3 presentó el mayor porcentaje de CCas comparado con los otros tres lotes tratados con DMH. El único grupo que presentó tumores benignos correspondió al lote 5 tratado con 17 inoculaciones de $\mathrm{DMH}+\mathrm{AcBu}$.

Frecuencia de los diferentes tipos histológicos. El lote DMH-17 presentó 166 tumores, de los cuales 132 fueron hallados macroscópicamente y 34 en el estudio microscópico, siendo todos malignos. El lote $\mathrm{AcBu}+\mathrm{DMH}-17$ registró 142 tumores, de los cuales 118 fueron hallados macroscópicamente y 24 en el estudio microscópico, correspondiendo a 2 tumores benignos y 140 tumores malignos. Los animales tratados con $\mathrm{AcBu}$ presentaron menor desarrollo de CCas al compararlos con los animales que no recibieron $\mathrm{AcBu}$. El lote $\mathrm{DMH}+\mathrm{AcBu}$ no presentó neoplasias de tipo $\mathrm{CMu}$, considerada de alto grado de malignidad. Con respecto a CMix y ACS, no se observaron diferencias entre ambos lotes con 17 inoculaciones. El lote DMH-5 presentó 63 tumores, 54 hallados macroscópicamente y 9 en el estudio microscópico, siendo todos malignos. El lote $\mathrm{AcBu}+\mathrm{DMH}-5$ presentó 54 tumores, 50 hallados macroscópicamente y 4 en el estudio microscópico, todos malignos. Los animales suplementados con AcBu

Tabla 3. Tipos histológicos de tumores registrados en ratas tratadas con DMH.

\begin{tabular}{lccccccc}
\hline \multicolumn{1}{c}{ lote } & AT & ATV & ACS & CCas & CMu & CMix & total \\
\hline $3(\mathrm{DMH}-17)$ & - & - & 132 & 22 & 2 & 10 & 166 \\
& & - & $79,52 \%$ & $13,25 \%$ & $1,20 \%$ & $6,02 \%$ & 3 \\
$4(\mathrm{DMH}-5)$ & 1 & - & $79,36 \%$ & $4,76 \%$ & $11,11 \%$ & $4,76 \%$ & 63 \\
$5(\mathrm{AcBu}+\mathrm{DMH}-17)$ & $0,7 \%$ & $0,7 \%$ & $88,02 \%$ & $2,82 \%$ & - & $7,75 \%$ & 142 \\
$6(\mathrm{AcBu}+\mathrm{DMH}-5)$ & - & - & $87,03 \%$ & $7,40 \%$ & - & $5,56 \%$ & 54 \\
total & 1 & 1 & 353 & 34 & - & 27 & 425 \\
\hline
\end{tabular}

AT: adenoma tubular, ATV: adenoma tubulo-velloso, ACS: adenocarcinoma simple, CCas: carcinoma de células en anillo de sello, CMu: carcinoma mucinoso, CMix: carcinoma mixto. 
presentaron menor número de neoplasias de tipo CCas, CMix y $\mathrm{CMu}$, independientemente del tratamiento con distintas dosis del cancerígeno.

Comportamiento infiltrativo de los distintos tipos histológicos. Casi todas las neoplasias malignas mostraron comportamiento infiltrativo en mayor o menor grado, presentándose la submucosa como el estrato más afectado (Figura 2). Por otra parte, un menor porcentaje de lesiones microscópicas se presentaron libres o sueltas en la luz del intestino no habiéndose encontrado su unión con la mucosa en los cortes seriados. De estas lesiones libres, 30 ACS correspondieron al lote 3; 15 ACS correspondieron al lote 4; 34 ACS y 1 CMix al lote 5; y 14 ACS al lote 6. Las lesiones que presentaban displasia severa y ocupaban la totalidad de la mucosa sin afectar la muscular de la mucosa se clasificaron como ACS in situ (Figura 2).

En el lote 3 el 14,70\% de los ACS comprometieron toda la pared del intestino, en el lote 4 el $14,28 \%$, en el lote 5 el 13,18\% y en el lote 6 el 12,12\%, no habiéndose observado diferencias significativas en cuanto al poder infiltrativo de este tipo tumoral en los diferentes lotes. El 100\% de los CMu del lote 3 infiltraron toda la pared del intestino, mientras que solo en el $26,08 \%$ de los CCas se observó el mismo comportamiento. En los lotes restantes de estos tipos tumorales no se encontró este alto grado de infiltración. En los lotes 3, 4, 5 y 6 los CMix se encontraron invadiendo toda la pared del intestino en porcentajes que no mostraron diferencias significativas entre ellos.

Estudio histoquímico. Mediante técnicas histoquímicas realizadas en los diferentes tipos de tumores se constató reacción negativa para las mucosustancias ácidas y neutras de manera pareja. Sin embargo, en CCas y $\mathrm{CMu}$ se registró intensa coloración positiva para ambos tipos de mucosustancias.

\section{DISCUSIÓN}

Análisis macroscópico. Todos los animales inducidos con DMH desarrollaron tumores independientemente de la dosis del cancerígeno y del tratamiento con AcBu. Sin embargo, el lote 3 (DMH-17) presentó una cantidad $24,24 \%$ más elevada de tumores detectados macroscópicamente que el lote 4 (DMH-5). Por otra parte el lote $5(\mathrm{AcBu}+\mathrm{DMH}-17)$ presentó $20,71 \%$ más tumores que el lote $6(\mathrm{AcBu}+\mathrm{DMH}-5)$. Estas observaciones indican que la mayor cantidad de inoculaciones del cancerígeno se correspondió con el mayor número de tumores desarrollados, independientemente del tiempo transcurrido desde las inoculaciones hasta el sacrificio. Tales resultados son coincidentes con los de otros investigadores que utilizaron diferentes dosis de $\mathrm{DMH}$, alcanzando mayor número de tumores en igual cantidad de tiempo con un número de inoculaciones superior a diez ${ }^{23}$, ya que a mayor numero de inoculaciones disminuye el período de latencia entre la primera inyección de DMH y con esto la aparición de tumores ${ }^{10}$. La cantidad de cinco inoculaciones realiza- das en nuestro trabajo produjo un número de tumores significativamente menor a los aparecidos con inoculaciones mayores a diez ${ }^{23} \mathrm{o}$ incluso 17 inoculaciones como las llevadas a cabo en nuestra experiencia, donde se observó la misma cantidad de tumores que en los trabajos con diez inoculaciones de DMH.

El efecto apoptótico del $\mathrm{AcBu}$ citado por algunos autores, que ocurriría por una vía independiente al p53 ${ }^{12,13}$, así como el efecto de inducción a la expresión de p21/WAF1, que da como resultado disminución de la proliferación celular e inducción de la diferenciación y apoptosis ${ }^{13}$, se manifestarían con disminución del tamaño y número de tumores. En esta experiencia los animales que recibieron suplementación con $\mathrm{AcBu}$ mostraron una leve disminución del número y tamaño de los tumores independientemente de las dosis del cancerígeno, con valores no significativos desde el punto de vista estadístico. En este sentido el lote 6 $(\mathrm{AcBu}+\mathrm{DMH}-5)$ presentó la mayor frecuencia de tumores pequeños, comprendidos en el rango entre $1 \mathrm{y}$ $10 \mathrm{~mm}$, indicando que el tamaño probablemente fue influenciado por el $\mathrm{AcBu}$, apoyando la teoría de inducción de apoptosis y diferenciación del AcBu sobre las neoplasias colónicas.

Independientemente del tratamiento con $\mathrm{AcBu}$, todos los animales de los lotes inoculados con el cancerígeno desarrollaron tumores de tipo polipoides, planos y ulceroinfiltrantes. No obstante, en los lotes 5 $(\mathrm{AcBu}+\mathrm{DMH}-17)$ y $6(\mathrm{AcBu}+\mathrm{DMH}-5)$ se observó un mayor porcentaje de tumores de tipo polipoides en relación a sus respectivos controles sin AcBu. Por otra parte el lote 3 (DMH-17) fue el que presentó mayor porcentaje de tumores planos, coincidiendo con el mayor número de inoculaciones del cancerígeno y sin la suplementación de AcBu. Este resultado podría estar indicando que a mayor dosis del cancerígeno existiría mayor desarrollo de un tipo tumoral de comportamiento más agresivo.

Considerando que los tumores de tipo plano son los que presentan mayor capacidad infiltrativa y que en

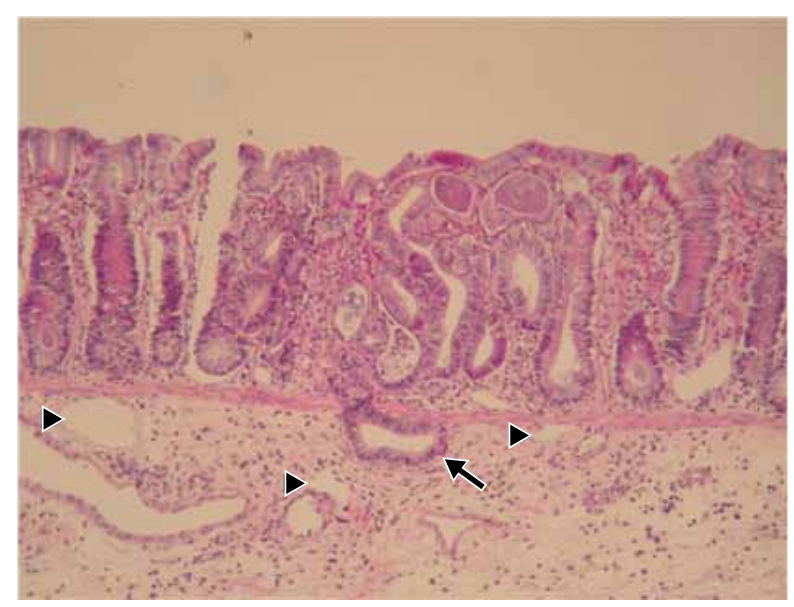

Figura 2. Adenocarcinoma invadiendo la submucosa a través de la muscular de la mucosa (flecha). La submucosa se encuentra edematizada, con vasos linfáticos dilatados (cabezas de flechas) (HE 10x). 
nuestra experiencia los lotes suplementados con $\mathrm{AcBu}$ fueron los que menos desarrollaron este tipo de tumor, se podría atribuir al AcBu la responsabilidad del menor desarrollo de un tipo tumoral más agresivo. Estos hallazgos coinciden con otros trabajos experimentales en ratas, realizados con leche de vaca y de búfala ${ }^{23}$. En estas experiencias, las ratas que recibieron dieta láctea presentaron también menor porcentaje de tumores planos. Tales resultados refrendan que diversos componentes de la dieta y específicamente en nuestro estudio el $\mathrm{AcBu}$, actuarían modificando el desarrollo de diferentes tipos de neoplasias del intestino inducidas experimentalmente.

Análisis microscópico. Resulta importante destacar que los lotes suplementados con $\mathrm{AcBu}$ presentaron menor desarrollo de tumores de comportamiento agresivo y caracterizados por su alto grado de malignidad, infiltración y metástasis. Se observó que el lote 3 (DMH-17) fue el que presentó mayor porcentaje de CCas $(13,86 \%)$ y el lote 4 (DMH-5) el mayor porcentaje de $\mathrm{CMu}(11,11 \%)$. Tanto CCas como CMu son tipos histológicos frecuentemente relacionados con tumores que macroscópicamente son planos y ulceroinfiltrantes. Contrariamente, los lotes suplementados con $\mathrm{AcBu}$ mostraron menor desarrollo de este tipo de neoplasias indicando que el $\mathrm{AcBu}$ tendría efectos sobre la diferenciación celular reduciendo la frecuencia de aparición de tumores de tipo indiferenciados.

Otro investigador trabajó con ratas a las cuales se les administró solamente DMH vía oral durante 12 semanas y registró mayor distribución de los tipos CCas, $\mathrm{CMu}$ y CMix en colon proximal y ciego ${ }^{16}$. Contrariamente, en nuestra experiencia estos tipos tumorales se distribuyeron de manera pareja entre los distintos segmentos del intestino grueso. En todos los animales de los lotes tratados con DMH los tumores se desarrollaron en los cuatro segmentos del intestino grueso, no habiéndose detectado diferencias significativas respecto a la localización en recto, colon distal y colon proximal independientemente del tratamiento con $\mathrm{AcBu}$. No obstante, el desarrollo de tumores en el ciego presentó ciertas características en los animales tratados con $\mathrm{AcBu}$ y con diferentes dosis de DMH.

El lote 3 (DMH-17) presentó mayor porcentaje de tumores con localización en ciego $(9,03 \%)$ en comparación con el lote 5 (AcBu+DMH-17) $(5,63 \%)$. Lo mismo sucedió entre los lotes 4 (DMH-5) $(15,87 \%)$ y 6 $(\mathrm{AcBu}+\mathrm{DMH}-5)$ (7,4 \%), con una mayor diferencia entre estos dos últimos dada posiblemente por la menor cantidad de inoculaciones con DMH. Esto indicaría que posiblemente, al aumentar la dosis del cancerígeno se disminuiría el posible efecto del AcBu sobre la distribución de los tumores en los distintos segmentos del intestino grueso. Este resultado es coincidente con la localización de tumores observada en seres humanos provenientes de poblaciones con alta incidencia de cáncer de colon, que presentan más afectadas las partes bajas del intestino grueso ${ }^{2}$. Lo mismo fue observado en estudios experimentales en ratas llevados a cabo con distintas dietas lácteas ${ }^{23}$.

Respecto al comportamiento infiltrativo de las neoplasias en las capas del intestino grueso, no se observaron diferencias significativas entre los lotes tratados con $\mathrm{AcBu}$, pero sí se presentaron diferencias entre lotes tratados únicamente con DMH. El lote 3 (DMH17), comparado con el lote 4 (DMH-5), presentó mayor proporción de tumores infiltrativos correspondiendo a adenocarcinomas simples y carcinomas mixtos. Estos resultados indican que el mayor número de inoculaciones de DMH aumentó no solo el número de tumores sino también la capacidad infiltrativa de los mismos.

Se ha sugerido que el butirato está implicado en la protección de colitis y cáncer colorrectal, pero que el efecto quimiopreventivo incluye diferencias entre el desarrollo in vivo e in vitro en el tiempo de administración del ácido butírico, así como en la cantidad y en la fuente de ácido butírico utilizada ${ }^{20}$. Estudios experimentales en ratones empleando tributirina como fuente de ácido butírico, no obtuvieron diferencias entre las áreas focales de displasia y tumores colónicos entre los animales tratados con esta sustancia y los controles ${ }^{8}$. Otros investigadores utilizaron butirato de sodio como fuente de ácido butírico administrado vía oral ${ }^{7}$ y vía intracecal ${ }^{18}$ en ratas. En algunas de estas experiencias dichas sustancias mostraron efectos anticancerígenos mientras que en otras no se reportó efecto alguno, considerándose que las sales de sodio favorecen el desarrollo de neoplasias colónicas inducidas químicamente ${ }^{8}$. En nuestro estudio no se constató un marcado efecto quimiopreventivo del AcBu sobre la frecuencia de presentación de neoplasias colónicas pero sí se verificaron efectos sobre el poder de diferenciación y desarrollo de los mismos, tales como presencia de tumores más pequeños, mayor número de tipo polipoides, considerados de comportamiento menos agresivo, menor número de tumores de tipo histológico CCas y $\mathrm{CMu}$ y menor número de neoplasias localizadas en ciego.

Estudio histoquímico. Las técnicas histoquímicas permitieron caracterizar los diferentes tipos histológicos de los tumores de acuerdo a la positividad a las coloraciones PAS y Alcian Blue. Sin embargo, no se observaron diferencias histoquímicas en los diferentes tumores de los lotes tratados con DMH independientemente de la suplementación con AcBu. Estos hallazgos coinciden con los observados en trabajos anteriores en los cuales los adenocarcinomas mostraron reducción de mucosustancias neutras y ácidas probablemente debido a la falta de diferenciación celular, mientras que los tumores con marcada producción de mucus como CCas y $\mathrm{CMu}$ fueron ricos en ambos tipos de mucinas ${ }^{23}$.

En base a los resultados obtenidos se puede concluir que con mayor estímulo cancerígeno se produce mayor número de neoplasias principalmente malignas, de comportamiento agresivo, mientras que con la suplementación de ácido butírico se reduce el número de neoplasias y el tamaño individual de cada una. Además, el ácido butírico modifica el comportamiento biológico de las neo- 
plasias al disminuir el desarrollo de tumores altamente malignos desde el punto de vista histológico y con capacidad de infiltrar y metastatizar. Del presente estudio puede inferirse que la manipulación de la dieta mediante la administración de ácido butírico se traduce en efectos específicos sobre el desarrollo de neoplasias colónicas.

\section{REFERENCIAS}

1. Basson MD, Emenaker MJ, Hong F. 1998. Differential modulation of human (Caco 2) colon cancer cell line phenotype by short chain fatty acids. Proc Soc Exp Biol Med 217: 476-483.

2. Bingham SA. 1996. Epidemiology and mechanisms relating diet to risk of colorectal cancer. Nutr Res Rev 9: 197239.

3. Boring CC. 1994. Cancer statistics. Cancer J Clin 44: 7.

4. Bravo A, Afonso JJ, Medina V, Pérez J, Lorenzo N, Fernández MV, González F. 2000. Butirato y carcinogénesis colorrectal. Cir Esp 68: 57-64.

5. Castro JA. 1986. Cáncer, alimentación y alimento. Epidemiología y etiología del cáncer de colon. Acta Bioq Clín Lat 17: 213-231.

6. Chen ZX, Breitman TR. 1994. Tributyrin: a prodrug of butyric acid for potential-clinical application in differentiation therapy. Cancer Res 54: 3494-3499.

7. D’Argenio G, Mazzacca G. 1999. Short chain fatty acids in the human colon. Relation to inflammatory bowel diseases and colon cancer. Adv Exp Med Biol 472: 149-158.

8. Deschner EE, Ruperto JF, Lupton JR, Newmark HL. 1990. Dietary butyrate (tributyrin) does not enhance AOMinduced tumorigenesis. Cancer Lett 52: 79-82.

9. Doll R. 1992. The lessons of life: keynote address to the nutrition and cancer conference. Cancer Res 52: 20242029.

10. Glauert HP, Weeks JA. 1989. Dose and time-response on colon carcinogenesis in Fischer-344 rats after a single dose of 1,2-dimethylhydrazine. Toxicol Lett 48: 283-287.

11. Haase P, Corven DM, Knowles JC. 1973. Evaluation of dimethylhydrazine induced tumors in mice as a model system for colorectal cancer. Br J Cancer 28: 530-543.

12. Haffner R, Oren M. 1995. Biochemical properties and biological effects of p53. Curr Opin Genet Dev 5: 84-90.

13. Hague A, Diaz GD, Hicks DJ, Krajewski S, Reed JC, Pereskeva C. 1997. Bcl-2 and bak may play a pivotal rol in sodium butyrate induced apoptosis in colonic epithelial cells: However overexpression of bcl-2 does not protect against bak mediated apoptosis. Int J Cancer 72: 898-905.
14. Keenan KP, Laroque P, Dixit R. 1998. Need for dietary control by caloric restriction in rodent toxicology and carcinogenicity studies. $J$ Toxicol Environ Health B 1: 135148 .

15. Lindström CG. 1978. Experimental colo-rectal tumours in the rat. Acta Pathol Microb Scandin Section A. Supplement $\mathrm{n}^{\mathrm{o}} 268$.

16. Martin MS. 1992. Experimental intestinal carcinogenesis. Cancer J 5: 5-10.

17. Matsui T, Yao T, Yao K, Takenaka K, Sakurai T, Iwashita A, Fuchigami T, Aoyagui K, Date H. 1996. Natural history of superficial depressed colorectal cancer: retrospective radiographic and histologic analysis. Radiology 201: 226-232

18. Medina V, Afonso JJ, Álvarez-Arguelles H, Hernández C, González F. 1998. Sodium butyrate inhibits carcinoma development in a 1,2-dimethylhydrazine induced rat colon cancer. J Parent Enter Nutr 22: 14-17.

19. Moonlenbeek C, Ruitenberg EJ. 1981. The 'Swiss roll': a simple technique for histological studies of the rodent intestine. Lab Anim 15: 57-59.

20. Nichenametla SN, South EH, Exon JH. 2004. Interaction of conjugated linoleic acid, sphingomyelin, and butyrate on formation of colonic aberrant crypt foci and immune functions in rats. $J$ Toxicol Environ Health Part $A$ 67: 469-481.

21. Parodi PW. 1996. Milk fat components: possible chemopreventive agents for cancer and other diseases. Austrian $J$ Dairy Tech 51: 24-32.

22. Pryde SE, Duncan SH, Hold GL, Stewart CS, Flint HJ. 2002. The microbiology of butyrate formation in the human colon. FEMS Microbiol Lett 217: 133-139.

23. Sánchez Negrette M, Montenegro MA, Catuogno MS, Lértora WJ, Guanziroli MC. 2007. Decrease of intestinal tumors induced by 1,2-dimethylhydrazine in rats fed with cow milk and buffalo milk. Biocell 31: 391-396.

24. Scheppach W, Bartram HP, Ritcher F. 1995. Role of short chain fatty acids in the prevention of colorectal cancer. Eur J Cancer 31A: 1077-1080.

25. Wiebecke B, Krey U, Loehrs U. 1973. Morphological and autoradiographical investigations on experimental carcinogenesis and polyps development in the intestinal tract of rats and mice. Virchows Arch Pathol Anat 360: 179-193.

26. Winneker RC, Tomkins M, Wesrenberg P. 1977. Tumors in hamsters. Exp Mol Pathol 27: 19-34.

27. Wynder EL, Shigematsu T. 1967. Environmental factors of cancer of the colon and rectum. Cancer 20: 1520-1561. 